Cahiers $d u$ MONDE RUSSE

\section{Cahiers du monde russe}

Russie - Empire russe - Union soviétique et États indépendants

$48 / 4 \mid 2007$

Varia

\title{
Rosalind P. Blakesley, Susan E. Reid, éds., Russian Art and the West
}

\author{
Gábor T. Rittersporn
}

\section{OpenEdition}

Journals

Édition électronique

URL : https://journals.openedition.org/monderusse/6136

DOI : 10.4000/monderusse.6136

ISSN : $1777-5388$

Éditeur

Éditions de l'EHESS

\section{Édition imprimée}

Date de publication : 2 décembre 2007

Pagination : 848-851

ISBN : 978-2-7132-2148-4

ISSN : $1252-6576$

Référence électronique

Gábor T. Rittersporn, « Rosalind P. Blakesley, Susan E. Reid, éds., Russian Art and the West », Cahiers du monde russe [En ligne], 48/4 | 2007, mis en ligne le 16 juin 2009, consulté le 03 septembre 2022. URL : http://journals.openedition.org/monderusse/6136 ; DOI : https://doi.org/10.4000/monderusse. 6136

Ce document a été généré automatiquement le 3 septembre 2022.

Tous droits réservés 


\title{
Rosalind P. Blakesley, Susan E. Reid, éds., Russian Art and the West
}

\author{
Gábor T. Rittersporn
}

\section{RÉFÉRENCE}

Rosalind P. BLAKESLEY, Susan E. REID, éds., Russian Art and the West. A Century of Dialogue in Painting, Architecture and the Decorative Arts. DeKalb, IL : Northern Illinois University Press, 2007, 246 p.

1 Le titre de ce volume a tout pour rebuter le lecteur potentiel. Il suggère des variations bien connues sur des thèmes comme symbolisme, cubisme, futurisme ou ce qu'on appelle " césannisme » en Russie, et sur l'émerveillement des artistes de l'Âge d'argent devant les tableaux français des collections de Sergej Ščukin et d'autres mécènes. De même, le titre ne mentionne pas l'époque soviétique à propos de laquelle il est convenu de déplorer que la grandeur de l'avant-garde et des influences occidentales ait cédé la place à la misère du grand renfermement et à de vaillantes tentatives de redécouvrir un riche héritage. Seul le lecteur patient qui s'aventure jusqu'au sous-titre risque de découvrir qu'il s'agit de cent ans de dialogues et non du sujet rebattu de l'apprentissage et de l'originalité des artistes russes.

2 C'est fort dommage. Car les auteurs de cet ouvrage collectif font un tour très intéressant des lieux et des milieux de rencontres, des ruptures et des rendez-vous manqués. Ils ne partent pas d'un concept des arts de l'Ouest et de la Russie qui seraient monolithiques et porteurs de valeurs en soi. Ils sont curieux de mondes créateurs multidimensionnels ainsi que d'un Occident et d'une URSS des arts qui, avant de devenir catégories géopolitiques, sont références et partenaires à la fois.

3 Rosalind P.Blakesley et Elizabeth Kridl Valkenier étudient deux moments des rapprochements et des éloignements $\mathrm{au} \mathrm{XIX}^{\mathrm{e}}$ et au début $\mathrm{du} \mathrm{Xx}^{\mathrm{e}}$ siècle. Blakesley décrit les efforts d'Aleksej Bogoljubov, animateur de la colonie parisienne des pensionnaires de l'Académie des arts de Pétersbourg, pour sensibiliser les jeunes Russes aux nouveaux 
courants en France et en Allemagne dans les années 1870. Il se heurta à la résistance de rivaux qui étaient à la recherche d'un style national. Cependant, l'impact de son travail de médiateur est indéniable.

4 Valkenier saisit l'instant où le rapprochement est devenu fait accompli. Malgré des débuts quelque peu non conformistes dans les années 1860, le mouvement des Peredvižniki (les Ambulants) est devenu, au cours des décennies suivantes, le défenseur officiel de l'orthodoxie nationale dans les arts. À la fin des années 1890, la revue Mir iskusstva rassemblait des jeunes artistes qui avaient peu de choses en commun avec les vénérables vieillards. Valkenier explique que le groupe, moins radical qu'on ne le croit, réussit à faire admettre l'ouverture sur l'Ouest. Son succès à l'étranger aboutit à intégrer l'art russe dans la modernité internationale.

5 Jane A. Sharp s'aventure sur les marches de l'Empire russe et de la Russie soviétique. Elle ouvre une perspective où Moscou représentait l'Ouest pour les artistes. C'était le cas de peintres géorgiens : sans renier l'héritage occidental, ils se réclamaient des traditions de leur pays. Ils avaient de bonnes raisons de se voir comme médiateurs entre cultures, assumant volontiers de s'égarer de temps à autre. En se tournant vers la Russie, Sharp prend l'exemple de Natal'ja Gončarova et décrit sa subtile synthèse d'éléments empruntés à l'iconographie orthodoxe, à des peintres comme Velasquez et Le Greco et à l'art oriental. Selon elle, pour les artistes et théoriciens de l'avant-garde russe, l'Occident n'était pas une source de vérités intangibles. Leurs ambitions étaient holistiques avant la lettre.

6 John McCannon prolonge la problématique de Sharp en explorant l'idéal de la divinité féminine dans l'œuvre de Nikolaj Roerich. Il le situe au carrefour des mondes du christianisme orthodoxe et catholique, des mythes slaves, nordiques et bouddhistes, de la vogue des Scythes, de l'univers sophiologique et théosophique. Les conceptions du peintre présentent de fortes affinités avec des idées que l'on retrouve dans la création d'une pléiade de ses contemporains. Elles s'accordent avec des thèmes qui apparaissent chez Solov'ev, Blok, Remizov, Gumilev, Belyj et bien d'autres. Il s'agit des éléments d'une imagerie eurasienne qui a formé un ensemble syncrétique chez Roerich.

7 Quatre chapitres se penchent sur l'époque soviétique. Deux concernent l'architecture, malheureusement non traitée pour la période prérévolutionnaire. Sona S. Hoisington évoque l'obsession soviétique des gratte-ciel. Tout a commencé par une visite aux ÉtatsUnis des architectes d'un Palais des soviets jamais construit. On voit l'idée fixe de la rivalité avec l'Amérique. Boris Iofan, le maître d'œuvre du palais, et ses collègues, s'inspirant de toutes les innovations d'outre-Atlantique, voulaient bâtir un géant qui devait évidemment dépasser l'Empire State Building. L'aventure allait échouer. Mais Iofan revint à New York en 1939 comme auteur du pavillon soviétique à l'Exposition universelle. Le projet renouait avec la manie de dépasser le rival yankee : son élément central était une tour de quinze étages. Ceci étant dit, le dynamisme du bâtiment contrastait avantageusement avec la masse statique du pavillon américain. Pourtant, la même année, une tour similaire de Viačeslav Oltarževskij à l'Exposition de l'agriculture à Moscou fut détruite avant même d'être achevée. Oltarževskij passa quelque temps en prison. Après sa libération, il soutenait que les gratte-ciel qu'on commençait à élever à la fin des années 1940 n'avaient rien à voir avec un quelconque modèle américain. Stalin, quant à lui, exigeait des bâtiments gigantesques, convaincu que les vainqueurs de la guerre devaient impressionner le monde. Peu importait la ressemblance au prototype 
d'outre-mer. Il fut virtuellement interdit de prononcer le mot « gratte-ciel », on créa un terme russe et l'affaire fut réglée.

8 La regrettée Catherine Cook et Susan E. Reid passent en revue la reprise des contacts entre les architectes soviétiques et leurs collègues occidentaux. Le processus a commencé à un moment critique où le gigantisme de l'époque stalinienne était rejeté et que les moyens étaient mobilisés pour améliorer quelque peu les conditions de vie de la population. L'URSS risquait de rester sans projets susceptibles d'émerveiller l'Ouest. Malgré tout, le grand programme de construction de logements produisit des solutions urbanistiques et architecturales qui reçurent des avis favorables à l'étranger. Les quartiers construits rappelaient les projets constructivistes de l'URSS des années 1920 bénéficièrent d'un dialogue entre architectes soviétiques et occidentaux. L'Exposition universelle de 1958 fut l'occasion privilégiée de faire montre du renouveau de l'architecture au pays des Soviets. Le pavillon de l'URSS fit bonne figure à Bruxelles.

9 Alison Hilton traite un sujet inhabituel: les résidus de l'impressionnisme dans la peinture du réalisme socialiste. Il n'y avait guère d'artistes qui envisagent de continuer à cultiver ce style après le début des années 1930. Les chantres de la nouvelle orthodoxie du réalisme socialiste rivalisaient pour le dénoncer comme bourgeois et formaliste. Mais des procédés typiques de l'impressionnisme enrichissaient des œuvres idéologiquement acceptables de peintres comme Igor' Grabar', Jurij Pimenov, Arkadij Plastov et Sergej Gerasimov. L'homonyme de ce dernier, Aleksandr Gerasimov, puissant président de l'Union des artistes, était le pourfendeur de tout ce qu'il convenait de taxer de formalisme. Il fallut attendre 1956 et le retour dans les salles des musées des tableaux des impressionnistes français, invisibles depuis 1947, pour que le style soit réhabilité.

Le chapitre de Susan E. Reid embrasse les années mouvementées qui vont du milieu des années 1950 à celui des années 1960. La période fut marquée par des tentatives de moderniser le réalisme socialiste, qui impliquaient un nouveau regard sur l'art étranger. Reid s'appuie sur une large documentation pour montrer les espoirs, les tiraillements, les recherches et les déceptions qui n'étaient pas toujours celles des rénovateurs. L'Exposition universelle de Bruxelles fut un cuisant échec pour les conservateurs car les organisateurs et l'opinion internationale ne voulaient pas reconnaitre l'art soviétique contemporain comme partie prenante de la modernité. Reid analyse quelques moments forts des batailles pour l'ouverture sur l'Occident : affrontements autour de l'exposition Picasso en 1956; rencontre mémorable à l'occasion du Festival international de la jeunesse en 1957 entre artistes et public soviétiques et représentants de courants d'avantgarde à Moscou. L'auteur montre comment la médiation est-allemande a légitimé le modernisme d'artistes comme Otto Dix, Käthe Kollwitz, Max Beckmann et d'autres grandes figures des premières décennies du $\mathrm{xx}^{\mathrm{e}}$ siècle. Elle souligne aussi l'influence des monumentalistes mexicains et des néoréalistes italiens ainsi que du concept d'art humaniste des marxistes révisionnistes. L'article explore des dimensions mal connues de l'histoire de l'art soviétique.

11 Il fallait s'attarder sur quelques chapitres de l'ouvrage pour encourager le lecteur potentiel à aller au-delà d'un titre légèrement trompeur. La qualité des études est certes quelque peu inégale. On peut regretter l'absence de tel ou tel sujet. Il n'en reste pas moins que le volume renouvelle notre perspective sur le dialogue et sur les querelles de l'art russe et soviétique avec l'Occident. 